\title{
Optical nonlinearity of organic dyes as studied by Z-scan and transient grating techniques
}

\author{
UMAKANTA TRIPATHY, R JUSTIN RAJESH, PREM B BISHT* and \\ A SUBRAHAMANYAM \\ Department of Physics, Indian Institute of Technology - Madras, \\ Chennai 600 036, India \\ e-mail: bisht@iitm.ac.in
}

\begin{abstract}
The excited state absorption cross-section of 5,5'-dichloro-11-diphenylamino-3,3'-diethyl-10,12-ethylinethiatricarbocyanine perchlorate (IR140) have been measured by using a single beam transmission technique. Z-scan experiments have been used to find out a few nonlinear parameters. The excited state relaxation times have also been measured by using laser induced transient grating (LITG) technique.
\end{abstract}

Keywords. Excited state absorption; Z-scan; nonlinear refractive index; excited state relaxation; transient grating.

\section{Introduction}

Following the absorption of the radiation the molecules can occupy the excited states. If the pump intensity is higher, then the excited molecule may absorb the light further within its fluorescence lifetime and attain a higher excited state; this phenomenon is referred to as nonlinear absorption. Measurement of nonlinear refractive index in the presence of nonlinear absorption can be done by a single beam transmission measurement method. These measurements can give the value of excited state absorption cross-section $\left(\sigma_{e s}\right)$ besides other nonlinear parameters. Hercher ${ }^{1}$ have obtained the value of $\sigma_{e s}$ for a thick saturable absorber. Recently $\mathrm{Gu}$ et al have obtained the $\sigma_{e s}$ value for Ni-azo chelate dye films by open aperture Z-scan technique. ${ }^{2}$ The existence of excited state absorption transitions limits the maximum transmission of organic dyes to a well-defined value of less than unity.

The fluorescence lifetime of IR140 have been reported by Ganikanov et $a l^{3}$. The absorption and fluorescence characteristics of laser dye IR140 were reported by Robert Gray $e t \mathrm{al}^{4}$ and the solvation dynamics of organic dye IR140 has been investigated using the transient grating technique by Vauthey et al by fs to ps time scale in a series of solvents. 5

The laser induced transient grating (LITG) technique is generally used to investigate the properties of optically excited materials. ${ }^{6-8}$ The excited state absorption cross sections are of prime importance when dealing with the diffraction efficiency of the LITG of the dyes. The present paper deals with the experiments and the theoretical calculation of $\sigma_{e s}$ and other photophysical parameters of IR140. Rhodamine $6 \mathrm{G}$ has also been used as a standard. The experiments are based on a single beam transmission measurement method for $\sigma_{e s}$ calculations. For nonlinear parameter estimation we have used the Z-scan

*For correspondence 
technique. The decay profiles have been measured by LITG method. The excited state relaxation times have been calculated by fitting the decay curve.

\section{Theory}

\subsection{Effects of nonlinearity}

For a fast third order nonlinearity the index of refraction $\eta$ is expressed in terms of nonlinear refractive indices $\boldsymbol{\eta}_{\mathfrak{k}}$ (esu) through ${ }^{9}$

$$
\eta=n_{0}+\eta_{2} / 2\left[|\vec{E}|^{2}\right]=n_{0}+\Delta n
$$

where $n_{0}$ is the linear refractive index, $\vec{E}$ is the peak electric field.

The amplitude and phase of the electric field as a function of distance $(z)$ are governed by (2) and (3)

$$
\frac{\mathrm{d} \Delta \phi}{\mathrm{d} z}=\Delta n(I) k
$$

and

$$
\frac{\mathrm{d} I}{\mathrm{~d} z}=-\alpha I-\sigma_{e s} N_{e s} I
$$

where $\mathrm{d} z$ is the differential element of depth in the sample and (3) contains both linear $(\alpha)$ and nonlinear $\left(\sigma_{e s} . N_{e s}\right)$ absorption terms. Solving the equations in case of a third order nonlinearity and neglecting nonlinear absorption the instantaneous on-axis phase shift at focus is defined as 9,10

$$
\Delta \phi_{0}(t)=k \cdot \Delta n_{0}(t) L_{\mathrm{eff}}
$$

where $L_{\text {eff }}=(1-\exp (-\alpha L)) / \alpha$ is the effective length of the sample, in which $L$ is the length of the sample. $\Delta v_{0}(t)$ is the change in refractive index which is dependent on the fluence. The time averaged $\left\langle\Delta \phi_{0}(t)\right\rangle$ is related to the time-averaged index change $\left\langle\Delta n_{0}(t)\right\rangle$ through (4) for transient effect.

The average value of the third order nonlinear refractive index $\left(\boldsymbol{\eta}_{\mathbf{R}}\right)$ can also be determined by 2,10

$$
\eta_{\mathfrak{R}}(\mathrm{esu})=\left[\left(\sqrt{ } 2 c n_{0} \lambda\right) /\left(80 \pi^{2} I_{0} L_{\mathrm{eff}}\right)\right] \Delta \phi_{0},
$$

where $I_{0}$ is the peak on axis irradiance at focus, $c$ is the velocity of light and $\lambda$ is the wavelength of the laser.

The on axis phase shift at the focus $\left(\Delta \phi_{0}\right)$ can be obtained from the numerical fitting of the closed aperture data with ${ }^{11}$

$$
T=1+\left[4 x /\left(x^{2}+9\right)\left(x^{2}+1\right)\right] \Delta \Phi_{0}-\left[2\left(x^{2}+3\right) /\left(x^{2}+9\right)\left(x^{2}+1\right)\right] \Delta \psi 0,
$$

Here $\Delta \psi_{0}$ is the on-axis phase shift due to nonlinear absorption and $x=z / z_{0}$, where $z_{0}$ is the Rayleigh range. The ratio of $\Delta \psi_{0}$ and $\Delta \phi_{0}$ is known as coupling factor ( $\left.\rho\right)$. It also 
relates the real and imaginary parts of third order nonlinear susceptibility. ${ }^{11}$ However, in the absence of the nonlinear absorption the second term in (6) can be neglected. ${ }^{11}$

\subsection{Excited state absorption cross-section}

The ground state absorption cross-section $\left(\sigma_{13}\right)$ can be calculated by using BeerLambert's law. The $\sigma_{e s}$ value can be determined with the help of three and four level energy systems given in figure $1 \mathrm{a}$ and $\mathrm{b}^{1}$ by varying the input intensity of the laser beam. The steady state transmission of a thick sample is given by

$$
\mathrm{d} I / \mathrm{d} z=-I(\alpha+\beta)=-I \cdot\left[\left(\boldsymbol{\alpha}_{\theta}+\beta_{0}\left(I / I_{s}\right)\right) /\left(1+I I_{s}\right)\right] .
$$

The solution to the differential equation (7) (at $z=0, I=I_{0}$ ) is given by

$$
\ln T-\ln T_{0}=\left(\gamma_{0}-1\right) \ln \left(\left(\gamma_{0}+I_{0} / I_{s}\right) /\left(\gamma_{0}+T I_{0} / I_{s}\right)\right),
$$

where $\gamma_{0}$ is defined as

$$
\gamma_{0} \equiv \alpha_{0} / \beta_{0} \equiv \sigma_{13} / \sigma_{e s}
$$

By fitting the experimental data (in the graph $T$ vs $I_{0} / I_{s}$ ) for the numerical solution of (8) by Newton-Ralphson method, we can find out the value of $\gamma_{0}$. By using the value of $\gamma_{0}$ and $\sigma_{13}$, we can calculate the value of $\sigma_{e s}$ from (9).

\subsection{Diffraction efficiency and excited state relaxation times}

Diffraction efficiency of the thin grating is experimentally measured by taking the ratio of the intensity of the diffracted signal to that of the transmitted intensity of one of the

(a)

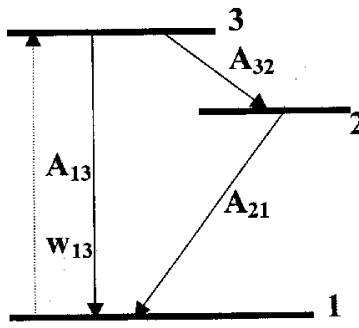

(b)

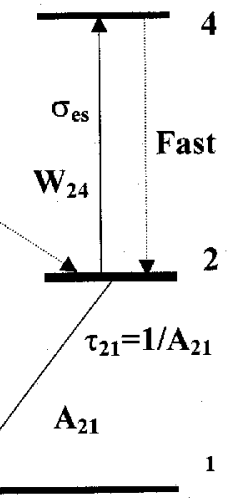

Figure 1. Energy schemes describing the excited state absorption cross-section with the help of three- (a) and four-level (b). $A_{21}$ is the transition probability from level 2 to level $1, W_{13}$ is the rate of absorption from level 1 to level 3 and $W_{24}$ is the rate of absorption from level 2 to level 4 . 
laser beams producing the grating. ${ }^{6}$ The time profiles obtained from the LITG experiment may contain several contributions such as the coherence spike, optical Kerr effect of the solvent (OKE), density phase grating, acoustic grating and relaxation times of the molecules. ${ }^{12-18}$ As a result the observed decay follows a complex equation. ${ }^{13,15}$ However, by excluding the longer part of the decay (fluorescence lifetime) as well as the coherence spike, the estimation of the excited state relaxation times can be done. ${ }^{19}$

\section{Experimental}

The experimental setup for the transmittance measurement as a function of incident intensity is shown in figure 2a. A frequency doubled mode-locked Nd:YAG laser (Continuum Model YG601, $35 \mathrm{ps}, 532 \mathrm{~nm}, 10 \mathrm{~Hz}$ ) was used as the light source. The radius of the beam waist $\left(\omega_{0}\right)$ was $4.1 \mathrm{~mm}$. The transmitted energy through the sample was measured by a PMT in the far field. ${ }^{1,20,21}$ For the measurement of other nonlinear parameters the Z-scan experimental setup is shown in figure 2(b). An Argon ion laser $(\lambda=514.5 \mathrm{~nm}$, power $=20 \mathrm{mw})$ was used as the light source. The measurement includes the transmittance measurement as a function of the position of the sample by using a positive lens having focal length $f=20 \mathrm{~cm}$. This measurement is known as Z-scan technique. The radius of the beam waist $\left(\omega_{0}\right)$ in this case was $68.6 \mu \mathrm{m}$ with a corresponding Rayleigh range of $2.87 \mathrm{~cm}$. The transmitted energy is measured by a PMT in the far field. Approximate care was taken against the surface imperfections in the sample that may lead to systematic transmittance trace change, which could mask the effect of nonlinear refraction. ${ }^{4,22}$ The experimental setup given in figure $2 \mathrm{~b}$ is known as closed aperture Z-scan setup, while an identical setup without the aperture is known as open aperture Zscan setup which gives $\sigma_{e s}$ value exclusively. The details of the experimental setup for creation and detection of the LITG are given elsewhere. ${ }^{19}$ The solvents viz., dimethyl sulphoxide (DMSO), methanol (MeOH), ethylene glycol (Et Gly) of spectrograde quality were used as received.
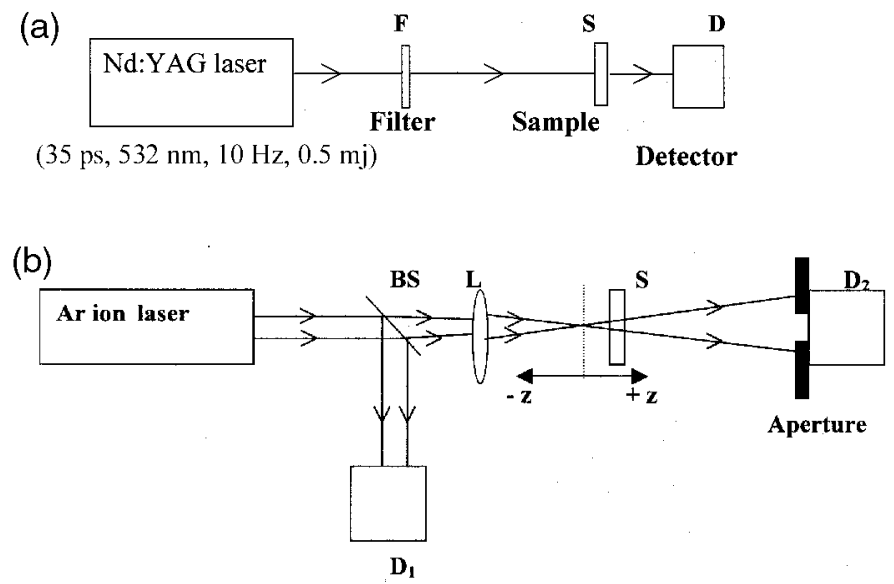

Figure 2. Experimental arrangement (a) for the measurement of excited state absorption cross-section: F - Filter, S - Sample, D - Detector; and (b) for Z-scan technique: $\mathrm{D}_{1}$ and $\mathrm{D}_{2}$ - Detectors, $\mathrm{BS}$ - Beam splitter, L - lens, $\mathrm{S}$ - sample and Zsample position. 


\section{Results and discussions}

\subsection{Measurement of $\sigma_{e s}$}

The photophysical parameters of the dyes are given in table 1. Figure 3 shows a result obtained from the energy dependence of the transmission. At lower intensities (region A),

Table 1. Photophysical parameters for laser dyes.

\begin{tabular}{|c|c|c|c|c|c|c|c|c|}
\hline \multirow{2}{*}{$\begin{array}{l}\text { Dyes and } \\
\text { solvents }\end{array}$} & \multicolumn{2}{|c|}{ Peak value (nm) } & \multirow{2}{*}{$\begin{array}{c}\sigma_{13} \\
\left.\times 10^{-16}\right) \\
\mathrm{cm}^{2}\end{array}$} & \multirow{2}{*}{$\begin{array}{c}\sigma_{e s} \\
\left(\times 10^{-18}\right) \\
\mathrm{cm}^{2}\end{array}$} & \multirow[b]{2}{*}{$\tau_{\mathrm{F}}$} & \multirow[b]{2}{*}{$\tau_{\text {or }}$} & \multirow{2}{*}{$\begin{array}{c}\text { Sign of } \\
\eta_{2}\end{array}$} & \multirow{2}{*}{$\begin{array}{l}\chi^{3} \\
\text { esu }\end{array}$} \\
\hline & $\lambda_{\mathrm{ABS}}$ & $\lambda_{\text {EMISS }}$ & & & & & & \\
\hline \multicolumn{9}{|l|}{$R 6 G$} \\
\hline $\begin{array}{l}\text { MeOH and } \\
\text { Et. Gly. }(1: 1) \\
\text { DMSO } \\
\text { PMMA }^{\text {a }}\end{array}$ & 530 & 562 & $1 \cdot 6$ & & $3.6 \pm 0.5 \mathrm{~ns}^{24}$ & $150 \pm 24 \mathrm{ps}$ & Negative & \\
\hline \multicolumn{9}{|l|}{ IR140 } \\
\hline $\begin{array}{l}\text { DMSO } \\
\mathrm{MeOH}\end{array}$ & $\begin{array}{l}820 \\
800^{4}\end{array}$ & $\begin{array}{l}837^{b} \\
830^{4}\end{array}$ & $24 \cdot 8$ & $3 \cdot 73$ & $231 \pm 64 \mathrm{ps}$ & & Negative $^{c}$ & d \\
\hline $\begin{array}{l}\text { Et. Gly } \\
\text { Et. Gly and } \\
\text { DMSO }(1: 1)\end{array}$ & & & & & $\begin{array}{c}165 \pm 5 \mathrm{ps}^{3} \\
240 \mathrm{ps}^{3}\end{array}$ & $288 \pm 8 \mathrm{fs}^{3}$ & & \\
\hline
\end{tabular}

${ }^{a}$ Polymethyl methacrylate (PMMA)

${ }^{\mathrm{b}}$ The solvent is $\mathrm{MeOH}+\mathrm{DMSO}(1: 1)$

${ }^{\mathrm{c}}$ The value of $\eta_{2} \approx-(3.73 \pm 0.06) \times 10^{-4}$ esu

${ }^{\mathrm{d}}$ The real value of $\chi^{(3)}$ i.e $\chi_{R}^{(3)}=-(1 \cdot 0 \pm 0.02) \times 10^{-12} \mathrm{~m}^{2} / \mathrm{V}^{2}$

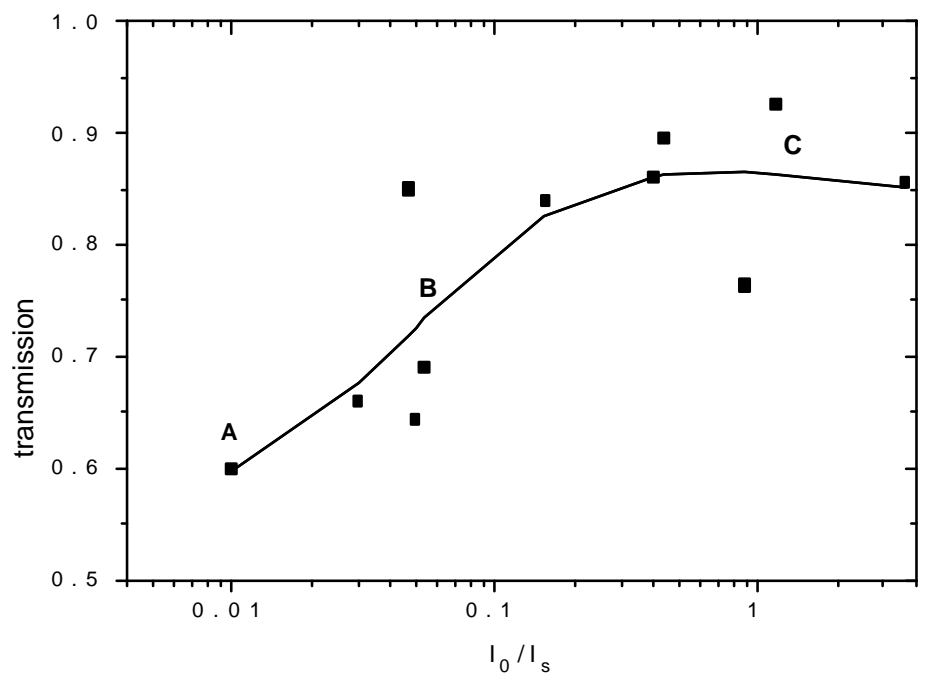

Figure 3. Plot of $I_{0} / I_{s}$ vs transmission intensity for IR140 in DMSO for the calculation of excited state absorption cross-section. The parameters are: Concentration is $2.5 \times 10^{-4} \mathrm{M}, \lambda=532 \mathrm{~nm}, \tau_{p}=35 \mathrm{ps}$ and $\sigma_{e s}=3.73 \times 10^{-18} \mathrm{~cm}^{2}$. 
the transmission shows low values corresponding to a small signal transmission $\left(T_{0}\right)$, followed by a region $\mathrm{B}$ where the transmission increases. In third region the transmission is high and approaches unity (region C). Region A is the linear absorption region, here the transmission follows the Beer's law, i.e. the effect of the absorptive transition between excited states is negligible. In region B as we increase the incident energy of laser pulse the molecules in the thermal equilibrium position of first excited state (level 2 of figure 1b) absorb the energy and go to the higher excited state (level 4 of figure 1(b)). Here the term $\sigma_{e s}$ comes in to picture. The region $C$ is known as residual absorption, ${ }^{1}$ which is a nonsaturable component of the absorption. The obtained transmission curve was simulated with the theoretical formulation developed in $\$ 2$, which gives the value of $\sigma_{e s}$ for this system.

\subsection{Measurement of $\left(\mathbf{\eta}_{\mathfrak{k}}\right)$}

In the Z-scan experiment the translation of the sample leads to change of the incident intensity falling on the sample, which results in a change in the refractive index $\left(\boldsymbol{\eta}_{\mathrm{R}}\right)$ of the sample. The sign as well as the magnitude of $\eta_{\mathrm{R}}$ for the dye IR140 can be determined by measuring the transmission through the circular aperture after the sample. ${ }^{22}$

Figure 4 shows the experimental result for IR140 in solvent DMSO obtained by closed aperture Z-scan technique. A prefocal transmittance maximum (peak) followed by a postfocal transmittance minimum (valley) in the Z-scan experiment is a signature of negative refractive nonlinearity. Following the same analogy, a positive nonlinear refraction gives rise to an opposite valley-peak configuration. The Z-scan signature for the organic dye

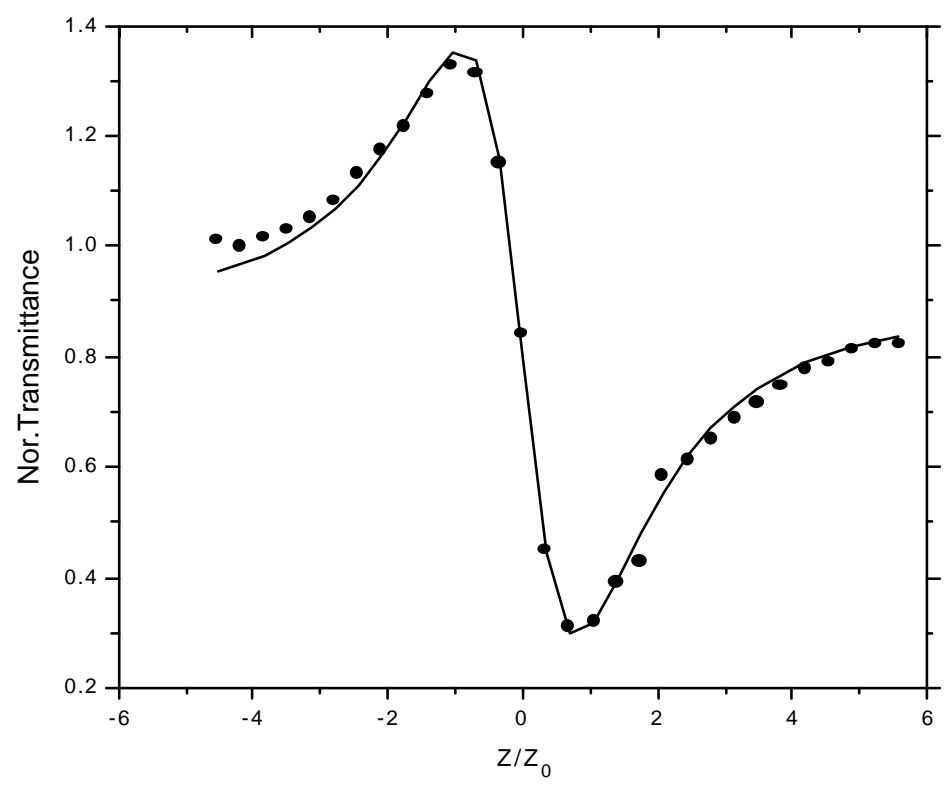

Figure 4. Plot for $z / z_{0}$ vs normalized transmittance for IR $140\left(1 \times 10^{-4} \mathrm{M}\right)$ in DMSO. The parameters are: $\lambda=514.5 \mathrm{~nm}$, Power $=20 \mathrm{mw}, \Delta T_{p-v}=1.02, \Delta \phi_{0}=$ $-(2.62 \pm 0.04)$ and the coupling factor $\rho=-(0.06 \pm 0.01)$ 


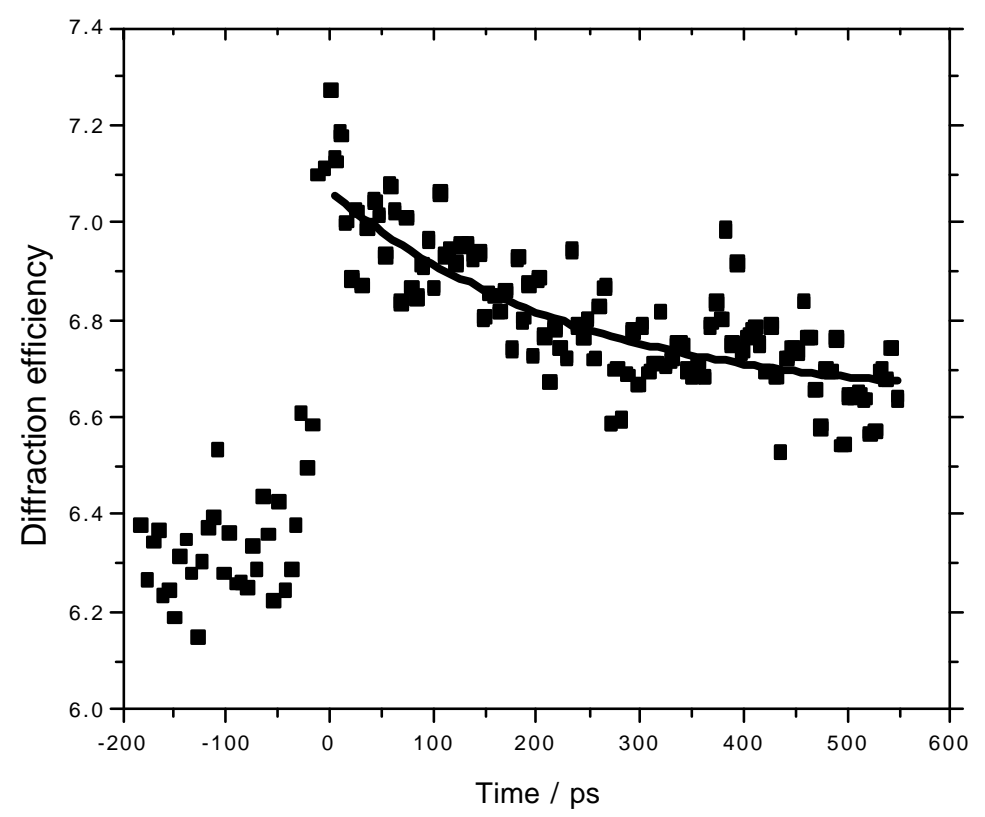

Figure 5. Decay curves for IR140 $\left(2.5 \times 10^{-4} \mathrm{M}\right)$ in DMSO. The fitted line for a single exponential decay function for a limited range is also shown. The parameters are: $\lambda=532 \mathrm{~nm}, \tau_{p}=35 \mathrm{ps}$ and $\tau_{F}=231 \pm 64 \mathrm{ps}$.

IR140 in DMSO shows negative refractive nonlinearity. The value of the transmission from peak to valley $\left(\Delta T_{p-v}\right)$ is 1.02 and the on axis phase shift at the focus $\left(\Delta \phi_{0}\right)$ is obtained as $-(2.62 \pm 0.04)$, which gives an index change of $\left\langle\Delta n_{0}\right\rangle=-(3.53 \pm 0.06) \times 10^{-4}$. This value of $\left\langle\Delta n_{0}\right\rangle$ corresponds to the value of $\eta_{\mathrm{R}} \approx-(3.73 \pm 0.06) \times 10^{-4}$ esu. The real part of the third order nonlinear susceptibility has a value of $\chi_{R}^{(3)}=-(1.0 \pm 0.02) \times$ $10^{-12} \mathrm{~m}^{2} / \mathrm{V}^{2}$. From the method described in ref. [11] we have obtained the value of $\rho$ as $-(0 \cdot 06 \pm 0 \cdot 01)$.

\subsection{Measurement of relaxation time}

The decay profiles of the grating formed by the pump beams at lower pump energy $(<0.14 \mathrm{~mJ})$ for the IR140 in DMSO is shown in figure 5. The observed decay profile shows a sharp peak at short time followed by a decaying component. The sharp peak at zero time is known as the coherence spike, which occurs in LITG due to the diffraction of one pump pulse from the grating formed between the probe pulse and the other pump pulse at time zero. The coherence spike appears only when the pump and the probe beams are derived from the same laser. A Gaussian profile with a half-width equal to that of the pump laser pulse accounts for this. ${ }^{15,16}$ The decay curves can have a varying nature depending upon the polarisation of the pump and probe beams, intensity of the incoming beams and wavelength of probe beam. By fitting the limited range of the decay curve with a single exponential function a rough estimate of the excited state relaxation time of the molecules in the solvent can be obtained. We obtained average value of $\tau_{\text {or }}$ as $150 \pm 24$ ps for R6G in DMSO, which is in close agreement with the value obtained from 
Debye-Stokes-Einstein ${ }^{23}$ hydrodynamic theory. By using the same experimental setup we obtained the value of the lifetime of the decay of IR140 in the solvent DMSO. The value of the decay constant is $231.8 \pm 64.8 \mathrm{ps}$ which is equal to the fluorescence lifetime of IR140. ${ }^{5}$ The orientational relaxation time of IR140 in solvent EtGly is $288 \pm 8 \mathrm{fs}^{5}$ which will be buried in the coherence spike in our case.

\section{Conclusions}

We have investigated the excited state absorption cross-section of the organic dye IR140 by a single beam transmittance measurement as a function of incident intensity. The nonlinear transmittance was found fluence dependent on picosecond time scale at $532 \mathrm{~nm}$. The calculated $\sigma_{e s}$ for IR140 in DMSO is $3.73 \times 10^{-18} \mathrm{~cm}^{2}$. The excited state relaxation time is DMSO was found to be $231 \pm 64$ ps. From Z-scan experiment, it is observed that IR140 has negative refractive nonlinearity.

\section{Acknowledgments}

We thank Council of Scientific and Industrial Research, New Delhi and Department of Science and Technology, New Delhi for financial assistance.

\section{References}

1. Hercher M 1967 App. Opt. 6947

2. Gu Y Z, Gan F X, Wang S Q and Xu H J 2001 Opt. Commun. 197501

3. Ganikhanov F, Hsieh J-M, Ci-Ling Pan 1995 Opt. Commun. 114289

4. Gray R, Walton D, Bikerton J, Richards P and Heptinstall J 1996 Dyes Pigments 30321

5. Gumy J C, Nicollet O and Vauthey E 1999 J. Phys. Chem. A103 10737

6. Eichler H J, Gunter P and Pohl D W 1986 Laser induced dynamic gratings (ed.) T Tamir (Berlin: Springer-Verlag) vol. 50

7. Bisht P B 2001 Res. Chem. Intermed. 27539

8. Rajesh R J, Bisht P B 2002 Chem. Phys. Lett. 357420

9. Bahae M S, Said A A and Van Stryland E W 1989 Opt. Lett. 14955

10. Bahae M S, Said A A, Wei T-H, Hagan D J and Van Stryland E W 1990 IEEE J. Quant. Electron. 26760

11. Liu X, Guo S, Wang H and Hou L 2001 Opt. Commun. 197431

12. Gumy J-C and Vauthey E 1997 J. Phys. Chem. A101 8575

13. Gumy J-C, Nicolet O and Vauthey E 1999 J. Phys. Chem. A103 10737

14. Terazima M 1998 Adv. Photochem. 24255

15. Degg F W D and Fayer M D 1989 J. Chem. Phys. 912269

16. Mayers A B, Hochstrasser R M 1986 IEEE J. Quant. Electron. 221482

17. Salcedo J R, Siegman A E, Dlott D D and Fayer M D 1978 Phys. Rev. Lett. 4131

18. Eichler H J, Langhans D and Massman F 1984 Opt. Commun. 50117

19. Rajesh R J, Natarajan T S and Bisht P B 2001 Indian J. Pure Appl. Phys. 39636

20. Hammand P R 1979 IEEE J. Quant. Electron. 15624

21. Falkenstein W, Penzkofer A and Kaiser W 1978 Opt. Commun. 27151

22. Gunter P 2000 Nonlinear optical effects and materials (ed.) P Gunter (Springer Verlag) ch. 2.5.2, vol. 72 , p. 106

23. Fleming GR 1986 Chemical applications of ultrafast spectroscopy (New York: Oxford University Press) ch. 6, p. 124

24. Bisht P B, Fukuda K and Hirayama S 1996 J. Chem. Phys. 1059349 